\title{
Ignorance is a bliss: mathematical structure of many-box models
}

Tomasz I. Tylec 1, a) and Marek Kuś ${ }^{2}$

1) Institute of Theoretical Physics and Astrophysics, Faculty of Mathematics, Physics and Informatics, University of Gdask, 80-308 Gdask

2) Center for Theoretical Physics, Polish Academy of Sciences, Aleja Lotników 32/46, 02-668 Warsaw, Poland

We show that the propositional system of a many-box model is always a setrepresentable effect algebra. In particular cases of 2-box and 1-box models it is an orthomodular poset and an orthomodular lattice respectively. We discuss the relation of the obtained results with the so-called Local Orthogonality principle. We argue that non-classical properties of box models are the result of a dual enrichment of the set of states caused by the impoverishment of the set of propositions. On the other hand, quantum mechanical models always have more propositions as well as more states than the classical ones. Consequently, we show that the box models cannot be considered as generalizations of quantum mechanical models and seeking for additional principles that could allow to "recover quantum correlations" in box models is, at least from the fundamental point of view, pointless.

\footnotetext{
a)Electronic mail: tomasz.tylec@ug.edu.pl
} 


\section{INTRODUCTION}

Consider the following simple model originated from Popescu and Rohrlich ${ }^{1}$ : a system consisting of $k$ "black-boxes", i.e. devices interacting with external world only by means of input and output signals, where both sets of admissible inputs and admissible outputs (which my vary depending on input), are finite. A state of such system, which in this paper we will call a PR-state (to honor Popescu and Rohrlich), is defined by probabilities $P\left(\alpha_{1} \alpha_{2} \ldots \alpha_{k} \mid a_{1} a_{2} \ldots a_{k}\right)$ of getting a particular tuple of outcomes $\left(\alpha_{1}, \alpha_{2}, \ldots, \alpha_{k}\right)$ given a tuple of inputs $\left(a_{1}, a_{2}, \ldots, a_{k}\right)$ that, apart from usual requirements of positivity and normalization, satisfy additionally the so-called no-signaling properties,

$$
\sum_{\alpha_{i}} P\left(\alpha_{1} \ldots \alpha_{i} \ldots \alpha_{k} \mid a_{1} \ldots a_{i} \ldots a_{k}\right)=\sum_{\beta_{i}} P\left(\alpha_{1} \ldots \beta_{i} \ldots \alpha_{k} \mid a_{1} \ldots b_{i} \ldots a_{k}\right)
$$

for all boxes enumerated by $i$. In plain words, these requirements express the fact changing of input for one box should not affect results, if we are not interested in the outcome that this very box provides. We call such system an $k$-box model. In more physical terms, we can think of a box as an experimental apparatus that can measure one observable from a specified finite set. The observables are labeled by an input values, so the input value chooses an observable to be measured, and the outcome of a measurement is returned on output. Then the $k$-box model is a set of $k$ such devices, each of them performing localized measurement.

Box models proved its usefulness in (quantum) information theory (cf. Refs. 29 and

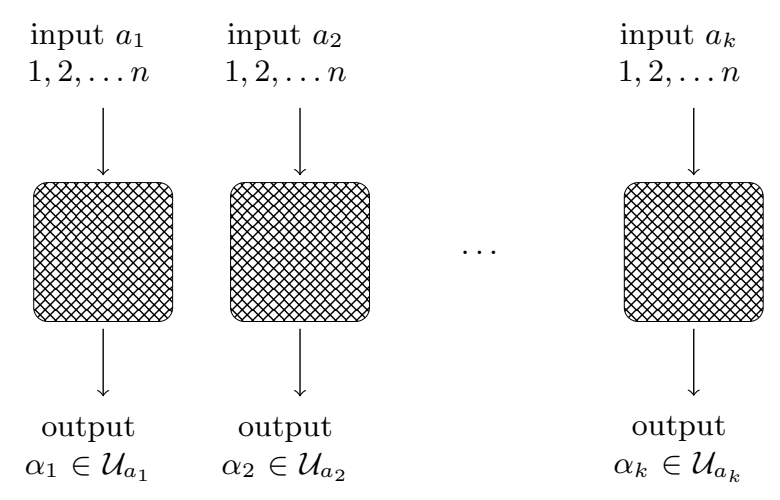

FIG. 1. A $k$-box model, i.e. system consisting of $k$ devices that produce output value upon providing it with an input. Devices are not connected with each other, thus can be placed in the space-like separated regions. 
many others, the original work of Popescu and Rohrlich has over 470 citation at the time of writing this paper). Most, if not all, use-cases for box models rely on the fact that some PRstates allow to obtain much stronger correlations between outputs of boxes than it is possible in quantum mechanics. This obviously resulted in numerous works where box models were applied to discussion of foundations of physics $10-16$.

However, various applications of box models are not accompanied with a rigorous and deep analysis of the mathematical structure that is inherently present in them. It is clear, that the $k$-box model defines a new probability theory that is claimed to be more general than the quantum probability theory. This paper is a continuation of our program of filling this gap and trying to understand properties of the box probability theory. Previously, we characterized the mathematical structure of 2-box model with a binary input and output 17 , followed by a general characterization of arbitrary 2-box models $\frac{18}{}$. Finally, in Ref. 19 we focused on description of how the mathematical structure of 2-box world arise from 1-box models. The present paper summarizes and extends all these works providing a general description of an arbitrary $k$-box model along with a discussion of how it emerges from structures of its components.

We work within the framework of the so-called quantum logics. This approach stems from the works of Birkhoff and von Neumann ${ }^{20}$ on foundations of quantum mechanics, later developed by Mackey ${ }^{21}$, Piron ${ }^{22}$, Ludwig ${ }^{23}$, among the others. Then the whole program of logic-based approach to foundations of physics slowly drifted apart from physics in the direction of pure mathematical study of various structures defined by a few physically inspired axioms. Nevertheless, this approach can be considered as a rigorous generalization of the Kolmogorov's probability theory competing with a more traditional quantum probability theory based on operator algebras. While the latter is unquestionably superior when it comes to analytical tools, the quantum logic approach, due to its simplicity and very fundamental nature, fits perfectly to rigorous study of box-models.

The paper is organized in the following way: firstly we briefly introduce some quantum logic structures and their properties that will be relevant for us. Then we discuss the structure of 1-box models and provide a general mathematical description of an arbitrary $k$-box model. The special case of 2-box models is discussed as an example. Finally, we discuss Local Orthogonality Principle ${ }^{14}, 24$ in the light of presented results. 


\section{LOGIC-BASED APPROACH}

We recall that a logic-based approach to physical models starts from the observation that we interact with a world by experiments and the most basic type of experiment is the so-called experimental question; it yields only two results: either "yes" or "no" (we will interchangeably use "true/false"). Since it is rather typical that a one physical property can be examined equivalently by a various experimental setups, we usually work on equivalence classes of experimental questions, which are called propositions (cf. Ref. 22 for more detailed discussion). The set of all propositions about a physical system has to satisfy certain properties. This leads to the following structures:

Definition 1 (see e.g. Ref. 25, Def. 1.2.1) An effect algebra $(E, \oplus, 0, \mathbb{1})$ is a set $E$ with a partially defined binary operation $\oplus$ and a distinguished elements 0 and $\mathbb{1}$ satisfying:

E1 whenever $p \oplus q$ is defined then $q \oplus p$ is defined and $p \oplus q=q \oplus p$,

E2 if $q \oplus r$ and $p \oplus(q \oplus r)$ are defined then $p \oplus q$ and $(p \oplus q) \oplus r$ are defined and

$$
p \oplus(q \oplus r)=(p \oplus q) \oplus r
$$

E3 for every $p$ there exists a unique $q$ such that $p \oplus q$ is defined and $p \oplus q=\mathbb{1}$,

E4 whenever $p \oplus \mathbb{1}$ is defined, $p=0$.

The interpretation is rather clear. The distinguished elements 0 and $\mathbb{1}$ stand for null and trivial propositions, i.e. ones represented by experimental questions that always yield "no" and "yes", respectively. From quantum mechanics we learned that logical "or" operator does not make sense for arbitrary pair of propositions, thus we implement "or" by a partially defined binary operation $\oplus$. E1 and E2 ensures that this "or" is good enough. We write $p \perp q$ whenever $p \oplus q$ exists and we say that $p, q$ are orthogonal or disjoint. Such propositions are exclusive: both cannot be simultaneously true. This justifies E4. Finally, for any proposition we can always consider its negation (we simply interchange answers). E3 ensures that there is a proposition corresponding to that negation. We can also introduce a partial order relation $p \leq q$ whenever there exists $r \in E$ such that $q=p \oplus r$ (see e.g. Ref. 25, Prop. 1.2.3). It is clear that $p \leq q$ means that whenever $p$ is true so is $q$. 
Element $p \in E$ is called an atom whenever $0 \leq q \leq p$ implies that either $q=0$ or $p=0$. An effect algebra is called atomic if for any $p \in E$ there exists an atom $a \leq p$ and atomistic if any element $p \in E$ is a $\oplus$-sum of atoms. Atoms represent the most elementary propositions and atomicity means that any proposition can be build from these elementary propositions assumption that is reasonable, at least for systems with finite number of degrees of freedom.

It is interesting to note, that although the E2 property allows us to drop parentheses in $\oplus$-sums with more than two elements, mutual orthogonality (so logical exclusiveness) of components does not guarantee that their $\oplus$-sum exists. The special case is:

Definition 2 (cf. Ref. 25, after Def. 1.5.4) An effect algebra $(E, \oplus, 0, \mathbb{1})$ satisfies a coherence law if

$$
p \perp q, q \perp r, r \perp p \Longrightarrow p \oplus q \oplus r \text { is defined, }
$$

in other words, whenever $\oplus$-sums exists for mutually orthogonal elements.

A typical example of an effect algebra is a set of all POVMs on a Hilbert space $\mathfrak{H}$, but it might be much more abstract and complicated structure though, like the set of compressions on a certain kind of Jordan algebras 26 .

When we focus on an order structure of propositions, instead of partially defined logical "or", following definition naturally emerges:

Definition 3 (cf. Ref. 27) An orthomodular poset (orthoposet in short) is a partially ordered set $L$ with a map ${ }^{\mathrm{c}}: L \rightarrow L$ such that

L1 there exists the greatest (denoted by $\mathbb{1}$ ) and the least (denoted by 0 ) element in $L$,

L2 map $p \mapsto p^{\mathrm{c}}$ is order reversing, i.e. $p \leq q$ implies that $q^{\mathrm{c}} \leq p^{\mathrm{c}}$,

L3 map $p \mapsto p^{\mathrm{c}}$ is idempotent, i.e. $\left(p^{\mathrm{c}}\right)^{\mathrm{c}}=p$,

L4 for a countable family $\left\{p_{i}\right\}$, s.t. $p_{i} \leq p_{j}^{\mathrm{c}}$ for $i \neq j$, the supremum $\bigvee\left\{p_{i}\right\}$ exists,

L5 if $p \leq q$ then $q=p \vee\left(q \wedge p^{\mathrm{c}}\right)$ (orthomodular law),

where $p \vee q$ is the least upper bound and $p \wedge q$ the greatest lower bound of $p$ and $q$.

Interpretation is again straightforward: $p \leq q$ means that whenever $p$ is true $q$ is also true; $p \mapsto p^{\mathrm{c}}$ maps proposition to its negation (justified by L2 and L3). If $p \leq q^{\mathrm{c}}$ then $p, q$ 
cannot both be true at the same time, so we write $p \perp q$ and call them orthogonal or disjoint like previously. L4 states then that for mutually exclusive propositions it should be possible to construct least proposition greater than all of them: the logical "or" over this set. Only L5 has no direct interpretation however has profound technical importance.

Since an effect algebra $E$ is a bounded partially ordered set (i.e. satisfies L1) and for $p \in E$ we might define $p^{c}$ to be equal to the unique element from E3 one might wonder when an effect algebra is actually an orthomodular poset:

Theorem 4 (see Ref. 25, Thm. 1.5.5) If an effect algebra $(E, \oplus, 0, \mathbb{1})$ satisfies a coherence law, then it is an orthomodular poset and $a \vee b=a \oplus b$ whenever $a \perp b$. Conversely, every orthomodular poset is an effect algebra satisfying coherence law.

Finally, we recall that an orthomodular lattice is an orthomodular poset in which each pair of elements $a, b$ has its supremum $a \vee b$ (or equivalently, infimum $a \wedge b$ ); a Boolean algebra is an orthomodular lattice in which distributive law is satisfied:

$$
a \vee(b \wedge c)=(a \vee b) \wedge(a \vee c)
$$

A typical examples are: the set of all projectors on a Hilbert space (an orthomodular lattice), the family of measurable sets on a measure space (Boolean algebra) and the family of all subsets of a finite set. Physical interpretation is obvious: projectors represent "yesno" measurements in quantum mechanics, while the latter two examples describe classical physical systems (the underlying set is a phase space of a system).

Another family of examples is represented by:

Definition 5 (see Ref. 27, Sec. 1.1) Let $\Delta$ be a family of subsets of some set $\Omega$ with partial order relation given by set inclusion and $A^{\mathrm{c}}=\Omega \backslash A$ satisfying:

C1 $\emptyset \in \Delta$,

C2 $A \in \Delta$ implies $\Omega \backslash A \in \Delta$,

C3 for any countable family $\left\{A_{i}\right\} \subset \Delta$ of mutually disjoint sets $\bigcup\left\{A_{i}\right\} \in \Delta$.

Then $(\Omega, \Delta)$ is called a concrete orthoposet. 
In particular, let $\Omega=\{1,2, \ldots 2 n\}$ and $\Delta$ be a family of subsets with even number of elements. Then $(\Omega, \Delta)$ is a concrete orthoposet which is orthomodular lattice for $n=2$ and Boolean algebra for $n=1$.

A physical system can be provided in different states. They are distinguished by different outcomes of experimental questions. Moreover, we do not require that an outcome of an experimental question will be the same each time we run it on a system in particular state. This leads to identification states with functions that assign to a proposition $p$ a value from unit interval $[0,1]$, which we will interpret as a probability of "true" answer for an experimental question representing proposition $p$; precisely:

Definition 6 (cf. Ref. 25, Def. 1.3.3) A state $\rho$ on an effect algebra $(E, \oplus, 0, \mathbb{1})$ is a map $\rho: E \rightarrow[0,1]$, s.t.

$\mathrm{S} 1 \rho(\mathbb{1})=1$,

S2 for a family $\left\{p_{i}\right\}_{i=1}^{n}$, s.t. $p_{1} \oplus p_{2} \oplus \cdots \oplus p_{n}$ is defined $\rho\left(\bigoplus_{i=1}^{n} p_{i}\right)=\sum_{i=1}^{n} p_{i}$.

We will denote by $\mathcal{S}(E)$ the set of all states on an effect algebra $E$. The same definition applies to orthoposets.

Remark 7 Typically $\sigma$-additive states are discussed, but all structures analyzed in the sequel have finite number of elements, thus we avoid unnecessary technicalities.

The set of admissible states $S$ of a physical system described by an effect algebra $E$ might be a proper subset of $\mathcal{S}(E)$. In that case however we require that the $S$ has enough states to distinguish different propositions by experiments, precisely:

$$
\forall \rho \in S, \rho(p)=\rho(q) \quad \Longleftrightarrow \quad p=q
$$

or even that the set propositions is order determining

$$
\forall \rho \in S, \rho(p) \leq \rho(q) \quad \Longleftrightarrow \quad p \leq q
$$

Definition 8 (see Ref. 25, Def. 1.10.1) Elements $p, q$ of an effect algebra $E$ are called compatible whenever there are $p^{\prime}, q^{\prime}, r \in E$ such that $p^{\prime} \oplus q^{\prime} \oplus r$ is defined and $p=p^{\prime} \oplus r$ and $q=q^{\prime} \oplus r$

Compatible elements can be described using classical, i.e. Kolmogorovian, probability (cf. Ref. 27, Thm. 1.3.23). 


\section{PROPOSITIONS IN BOX MODELS}

We will follow the standard approach to logic-based description of physical systems (see e.g. Ref. 21 and 22 for more detailed discussion). We start by describing the set of propositions of a single box model. Let us fix notation: input values will be enumerated $1,2, \ldots, N$, and the set of outcomes for the input $i$ will be denoted by $\mathcal{U}_{i}$ (we remind that all these sets are finite). Clearly

"does an input value $a$ result in an output from $\mathcal{A}$ ?"

where $\mathcal{A}$ is a subset of all admissible outputs for input $a$ is a good question about a one box system. We will denote by

$$
[a \in \mathcal{A}] \text { or }[a \alpha] \text { if } A=\{\alpha\}
$$

the proposition represented by that question. Before we dwell into analysis of the structure of the set of all propositions, let us observe that any PR-state $P$ on a 1-box model should define a proper state $\rho_{P}$ on the structure of propositions. In particular:

$$
\begin{aligned}
\rho_{P}([a \alpha]) & =P(\alpha \mid a), \\
\rho_{P}([a \in \mathcal{A}]) & =\sum_{\alpha \in \mathcal{A}} P(\alpha \mid a) .
\end{aligned}
$$

Since we do not restrict PR-states anyhow (no-signaling is trivial condition for 1-box), we immediately get that

$$
[a \in \mathcal{A}] \leq[b \in \mathcal{B}] \quad \Longleftrightarrow \quad\left\{\begin{array}{l}
\mathcal{B}=\mathcal{U}_{b}, \\
\mathcal{A}=\emptyset, \\
a=b \text { and } \mathcal{A} \subset \mathcal{B}
\end{array}\right.
$$

The special case $[a \in \emptyset]$ represents the null proposition: the one that is always false. Such a proposition is trivially in $\leq$ relation with any other proposition. Moreover $[a \in \emptyset]=[b \in \emptyset]$, since an experimental question that always results in the "false" answer is in both equivalence classes. Similarly $\left[b \in \mathcal{U}_{b}\right]$ represents a trivial proposition that is always true. Clearly it is in $\leq$ relation only with itself and $\left[a \in \mathcal{U}_{a}\right]=\left[b \in \mathcal{U}_{b}\right]$

The set of all propositions $\mathfrak{B}$ about a 1-box model apart from elements of the form $[a \in \mathcal{A}]$ 
should also contain their formal $\oplus$-sums

$$
[a \in \mathcal{A}] \oplus[b \in \mathcal{B}] \text { whenever } \rho_{P}([a \in \mathcal{A}])+\rho_{P}([b \in \mathcal{B}]) \leq 1,
$$

so that

$$
\rho_{P}([a \in \mathcal{A}]) \oplus \rho_{P}([b \in \mathcal{B}])=\rho_{P}([a \in \mathcal{A}])+\rho_{P}([b \in \mathcal{B}]) .
$$

Again, since there are no restrictions on $P$ other than positivity and normalization,

$$
[a \in \mathcal{A}] \oplus[b \in \mathcal{B}] \text { is defined } \quad \Longleftrightarrow \quad a=b \text { and } \mathcal{A} \cap \mathcal{B}=\emptyset \text {. }
$$

It follows then that

$$
[a \in \mathcal{A}]=\bigoplus_{\alpha \in \mathcal{A}}[a \alpha] \quad \text { and } \quad[a \in \mathcal{A}] \oplus\left[a \in \mathcal{U}_{a} \backslash \mathcal{A}\right]=\left[a \in \mathcal{U}_{a}\right]
$$

and

$$
[a \in \mathcal{A}] \oplus\left[a \in \mathcal{U}_{a} \mathcal{A}\right]=\left[a \in \mathcal{U}_{a}\right]
$$

Consequently, one immediately gets that the set

$$
\mathfrak{B}=\left\{[a \in \mathcal{A}] \mid \mathcal{A} \subset \mathcal{U}_{a} \text { and } a=1, \ldots, N\right\}
$$

of all propositions on a 1-box model, with the relation $\leq$ and the map

$$
[a \in A]^{\mathrm{c}}=\left[a \in \mathcal{U}_{a} \backslash \mathcal{A}\right]
$$

is a concrete orthomodular lattice (cf. Refs. 17 and 18). Denote by

$$
\Gamma(\mathfrak{B})=\mathcal{U}_{1} \times \mathcal{U}_{2} \times \cdots \times \mathcal{U}_{n}
$$

the phase space associated with a truly classical 1-box model (where a point of a phase space contains information about output the box will give for any input value). To each proposition $[a \in \mathcal{A}]$ we assign a subset of $\Gamma$

$$
\mathcal{U}_{1} \times \cdots \times \mathcal{U}_{a-1} \times \mathcal{A} \times \mathcal{U}_{a+1} \times \cdots \times \mathcal{U}_{n}
$$


All such subsets form a concrete logic and the order relation agrees with the order on $[a \in \mathcal{A}]$. Consequently, we can identify propositions of the 1-box model with the subsets of above form. Finally, let use remark that the logic $\mathfrak{B}$ is atomistic and elements $[a \alpha] \equiv[a \in\{\alpha\}]$ are atoms in $\mathfrak{B}$.

Example 9 Let us denote by $\mathfrak{B}_{\text {BIN }}$ a concrete logic of a 1-box model with binary input and output. Clearly $\Gamma\left(\mathfrak{B}_{\mathrm{BIN}}\right) \equiv \Gamma_{\mathrm{BIN}}=\{0,1\} \times\{0,1\}$. For readability, let us denote the input value 0 by $x$ and the input value 1 by $y$. The logic consists of 6 elements:

$$
\begin{array}{rlr}
\emptyset, & \{0\} \times\{0,1\} \equiv[x 0], \quad\{1\} \times\{0,1\} \equiv[x 1], \\
\{0,1\} \times\{0\} \equiv[y 0], & \{0,1\} \times\{1\} \equiv[y 1], & \Gamma_{2}
\end{array}
$$

Now let us discuss the set of propositions of a $k$-box model. We will denote it by $\mathfrak{B}^{\otimes k}$, however the use of $\otimes$ symbol should not be linked with the notion of tensor product, but rather a traditional way of indicating composite systems in physics.

Theorem 10 Let $(\Gamma, \mathfrak{B})$ be a concrete logic of a single box. Propositions of a $k$-box model $\mathfrak{B}^{\otimes k}$ are described by the concrete effect algebra of subsets of $\Gamma^{k}$ generated by

$$
\mathcal{A}=\left\{\left[a_{1} \alpha_{1}\right] \times \cdots \times\left[a_{k} \alpha_{k}\right] \mid a_{i}=1, \ldots, n ; \alpha_{i} \in \mathcal{U}_{a_{i}}\right\}
$$

where $p \oplus q$ is defined whenever $p \cap q=\emptyset$ and $\Gamma^{k} \backslash(p \cup q)$ can be decomposed into the union of mutually disjoint elements from $\mathcal{A}$. In that case, $p \oplus q=p \cup q$.

Proof It is straightforward to check that $\mathfrak{B}^{\otimes k}$ is an effect algebra. We need to show that elements of $\mathfrak{B}^{\otimes k}$ can be identified with propositions of a $k$-box model representing questions

We have already shown how propositions of a 1-box model can be encoded in subsets of $\Gamma$. Moreover, any $k$-tuple $\left(q_{1}, \ldots, q_{k}\right)$ of propositions of a 1 -box models $\mathfrak{B}$ is a proposition on a $k$-box model represented by the experimental question

does for all $i, q_{i}$ is true for the $i$-th box?

Thus, without loss of generality we can encode any such $k$-tuple as a Cartesian product $q_{1} \times \cdots \times q_{k}$. 
Observe now that the only subsets $q \in \mathfrak{B}^{\otimes k}$ of $\Gamma^{k}$ that have non-unique decomposition into elements of $\mathcal{A}$ are of the form

$$
\mathcal{A}_{1} \times \mathcal{A}_{2} \times \cdots \times \Gamma \times \ldots \mathcal{A}_{k}
$$

This remark allows us to extend an arbitrary PR-state $P$ to a we well defined state $\rho_{P}$ on $\mathfrak{B}^{\otimes k}$ by

$$
\begin{aligned}
\rho_{P}\left(\left[a_{1} \alpha_{1}, \ldots, a_{k} \alpha_{k}\right]\right) & =P\left(\alpha_{1}, \ldots, \alpha_{k} \mid a_{1}, \ldots, a_{k}\right), \\
\rho_{P}\left(q_{1} \oplus \cdots \oplus q_{n}\right) & =\sum_{i=1}^{n} \rho_{P}\left(q_{i}\right),
\end{aligned}
$$

where $q_{i} \in \mathcal{A}$ and Eq. (6) is not ambiguous thanks to the no-signaling property of PR-states.

Conversely, any state $\rho$ on $\mathfrak{B}^{\otimes k}$ satisfies a no-signaling property, thus we can assign a PR-state $P_{\rho}$ to it by

$$
P_{\rho}\left(\alpha_{1} \ldots \alpha_{k} \mid a_{1} \ldots a_{k}\right)=\rho\left(\left[a_{1} \alpha_{1}, \ldots, a_{k} \alpha_{k}\right]\right)
$$

To sum up, the structure of $\mathfrak{B}^{\otimes k}$ contains all most elementary propositions of a $k$-box model as an atoms, $\oplus$-sums of them, and the set of PR-states and states on $\mathfrak{B}^{\otimes k}$ coincide. This suffices to interpret $\mathfrak{B}^{\otimes k}$ as an effect algebra of propositions of a $k$-box model.

Remark 11 From the operational point of view, whenever:

$$
\rho_{p}\left(q_{1}\right) \oplus \cdots \oplus \rho_{P}\left(q_{n}\right) \leq 1, \quad \forall P, \quad q_{i} \in \mathcal{A}
$$

$q_{1} \oplus \cdots \oplus q_{n}$ should be defined. It is not clear that it implies that $\Gamma^{k} \backslash\left(q_{1} \cup \cdots \cup q_{n}\right)$ can be expressed as a sum of mutually disjoint elements of $\mathcal{A}$. However, if it could not, then adding such elements to $\mathfrak{B}^{\otimes k}$ would result in enlargement of the set of atoms, what would be operationally hard to interpret.

Elements of $\mathcal{A}$ are atoms of the effect algebra $\mathfrak{B}^{\otimes k}$ and it is clear that experimental questions that are representing them are the most elementary on a $k$-box model. To simplify our notation, we will write

$$
\left(\left[a_{1} \in \mathcal{A}_{1}\right],\left[a_{2} \in \mathcal{A}_{2}\right], \ldots,\left[a_{k} \in \mathcal{A}_{k}\right]\right) \equiv\left[a_{1} \in \mathcal{A}_{1}\right] \times \cdots \times\left[a_{k} \in \mathcal{A}_{k}\right] \equiv\left[a_{1} \in \mathcal{A}_{1} \ldots a_{k} \in \mathcal{A}_{k}\right]
$$


Elements of the form

$$
\left[\mathbb{1} a_{2} \in \mathcal{A}_{2} \ldots a_{k} \in \mathcal{A}_{k}\right]
$$

will be called localized in the boxes $\{2, \ldots, k\}$. Analogously we define propositions localized in an arbitrary subset of boxes; in particular, we say that

$$
[\mathbb{1} \mathbb{1} \ldots \underbrace{a \in \mathcal{A}}_{i \text {-th }} \ldots \mathbb{1}]
$$

is localized in the $i$-th box.

In order to interpret a $k$-box model as a composite system of $k$ separate boxes (that could be put in spacelike separate regions of a spacetime), we require that propositions localized in a different subsets of boxes are compatible. It is easy to see that indeed this is the case. Consider

$$
[\mathbb{1} \mathbb{1} \ldots \underbrace{a \in \mathcal{A}}_{i \text {-th }} \ldots \mathbb{1}] \text { and }[\mathbb{1} \mathbb{1} \ldots \underbrace{b \in \mathcal{B}}_{j \text {-th }} \ldots \mathbb{1}]
$$

Then

$$
[\mathbb{1} \mathbb{1} \ldots \underbrace{a \in \mathcal{A}}_{i \text {-th }} \ldots \mathbb{1}]=\bigoplus_{\beta \in \mathcal{U}_{b}}[\mathbb{1} \mathbb{1} \ldots \underbrace{b \beta}_{j \text {-th }} \ldots \underbrace{a \in \mathcal{A}}_{i \text {-th }} \ldots \mathbb{1}]
$$

and

$$
[\mathbb{1} \mathbb{1} \ldots \underbrace{b \in \mathcal{B}}_{j \text {-th }} \ldots \mathbb{1}]=\bigoplus_{\alpha \in \mathcal{U}_{a}}[\mathbb{1} \mathbb{1} \ldots \underbrace{b \in \mathcal{B}}_{j \text {-th }} \ldots \underbrace{a \alpha}_{i \text {-th }} \ldots \mathbb{1}]
$$

so the requirements of Def. 8 are clearly satisfied.

In Ref. 18 we constructed a propositional system of an arbitrary 2-box model in a similar fashion as in Thm. 10. It was a concrete orthomodular poset generated as a sublogic of Boolean algebra of subsets of $\Gamma \times \Gamma$ by the set $\mathcal{A}$, the same as in Thm. 10. It follows from the Lemma 11 of Ref. 18 that both constructions coincide. However, $\mathfrak{B}^{\otimes k}$ is not an orthomodular poset in general, as the following example shows.

Example 12 Consider a 3-box model described by $\mathfrak{B}_{\mathrm{BIN}}^{\otimes 3}$. It is known $\frac{14,28}{2}$ that there exists a PR-state $P$ such that

$$
P([x 0 x 0 x 0])+P([x 1 y 1 y 0])+P([y 0 x 1 y 1])+P([y 1 y 0 x 1])=\frac{4}{3}>1 .
$$


However all sets $[x 0 x 0 x 0],[x 1 y 1 y 0],[x 0 x 1 y 1]$ and $[y 1 y 0 x 1]$ are mutually disjoint. Actually, we can show that $[x 0 x 0 x 0] \oplus[x 1 y 1 y 0] \oplus[y 0 x 1 y 1],[y 0 x 1 y 1] \oplus[y 1 y 0 x 1]$ and $[x 0 x 0 x 0] \oplus$ $[x 1 y 1 y 0] \oplus[y 1 y 0 x 1]$ exist but $[x 0 x 0 x 0] \oplus[x 1 y 1 y 0] \oplus[y 0 x 1 y 1] \oplus[y 1 y 0 x 1]$ does not, so $\mathfrak{B}_{\text {BIN }}^{\otimes 3}$ cannot be organized into an orthomodular poset (cf. Def. 2).

The effect algebra $\mathfrak{B}_{\text {BIN }}^{\otimes 3}$ can be constructed explicitly. It has 28886 elements and $4^{3}=64$ atoms.

Consequently we proved:

Theorem 13 The propositional system of a $k$-box model is an orthomodular lattice for $k=1$ and orthomodular poset for $k=2$; otherwise it is an effect algebra.

An important property of composed systems is that the order of composition is irrelevant. This motivates the following definition:

Definition 14 Let $\left(\Gamma_{1}, \mathfrak{B}_{1}\right),\left(\Gamma_{2}, \mathfrak{B}_{2}\right)$ be atomistic concrete effect algebras. The box-product of $\mathfrak{B}_{1}$ and $\mathfrak{B}_{2}$ is defined as a concrete effect algebra $\left(\Gamma_{1} \times \Gamma_{2}, \mathfrak{B}_{1} \otimes \mathfrak{B}_{2}\right)$ where $\mathfrak{B}_{1} \otimes \mathfrak{B}_{2}$ is generated from the set

$$
\mathcal{A}=\left\{a \times b \mid a \in \operatorname{Atoms}\left(\mathfrak{B}_{1}\right), b \in \operatorname{Atoms}\left(\mathfrak{B}_{2}\right)\right\}
$$

by a partially defined binary operation

$$
p \oplus q= \begin{cases}p \cup q & \text { if } p \cap q=\emptyset \text { and } \Gamma_{1} \times \Gamma_{2} \backslash(p \cup q) \text { can be decomposed into } \\ & \text { a sum of mutually disjoint sets from } \mathcal{A} \\ \text { not defined } & \text { otherwise. }\end{cases}
$$

Remark 15 The box product is associative.

Proof Since the Cartesian product is associative we have that

$$
\begin{gathered}
\left\{a \times(b \times c) \mid a \in \operatorname{Atoms}\left(\mathfrak{B}_{1}\right), b \in \operatorname{Atoms}\left(\mathfrak{B}_{2}\right), c \in\left(\operatorname{Atoms} B_{3}\right)\right\}= \\
\left.\{(a \times b) \times c) \mid a \in \operatorname{Atoms}\left(\mathfrak{B}_{1}\right), b \in \operatorname{Atoms}\left(\mathfrak{B}_{2}\right), c \in\left(\operatorname{Atoms} B_{3}\right)\right\}
\end{gathered}
$$

and $\Gamma_{1} \times\left(\Gamma_{2} \times \Gamma_{3}\right)=\left(\Gamma_{1} \times \Gamma_{2}\right) \times \Gamma_{3}$. Consequently, the construction gives precisely the same elements in $\mathfrak{B}_{1} \otimes\left(\mathfrak{B}_{2} \otimes \mathfrak{B}_{3}\right)$ as in $\left(\mathfrak{B}_{1} \otimes \mathfrak{B}_{2}\right) \otimes \mathfrak{B}_{3}$ 
In other words, the propositional system of a $k$-box model if a $k$-fold box product $\mathfrak{B}^{\bowtie k}$ of 1-box propositional models. The set of PR-states coincide with the set of all states on this effect algebra.

\section{RELATION TO LOCAL ORTHOGONALITY PRINCIPLE}

Presented results shed new light on the recently proposed Local Orthogonality Principle $\underline{14}, 24$. In short, it puts additional restriction on the set of allowed correlations in the box models, i.e. on the set of admissible PR-states. Quoting Ref. $\stackrel{24}{ }$, it is phrased in the following way:

Definition 16 Consider a $k$-box model. An event consists of the $k$-tuple of inputs $a_{1}, \ldots, a_{k}$ and the corresponding $k$-tuple of outputs $\alpha_{1}, \ldots, \alpha_{k}$. Two events are orthogonal whenever at least one of the inputs coincides in two events but the corresponding output is different. Any set of mutually orthogonal events defines an Local Orthogonality (LO) inequality by requiring that the sum of probabilities for such set of events is less than or equal to 1 .

In the notation adopted in this paper, an event is an atom $\left[a_{1} \alpha_{1} \ldots a_{k} \alpha_{k}\right]$ of an effect algebra of a box model. Two events $\left[a_{1} \alpha_{1} \ldots a_{k} \alpha_{k}\right]$ and $\left[b_{1} \beta_{1} \ldots b_{k} \beta_{k}\right]$ are orthogonal if and only if

$$
\left[a_{1} \alpha_{1} \ldots a_{k} \alpha_{k}\right] \cap\left[b_{1} \beta_{1} \ldots b_{k} \beta_{k}\right]=\emptyset
$$

Finally, the Local Orthogonality inequality for $n$ mutually orthogonal events $q_{1}, \ldots, q_{n}$ is equivalent to the statement that for any state $\rho$ on an effect algebra of box model fulfills

$$
\rho\left(q_{1}\right)+\rho\left(q_{2}\right)+\cdots+\rho\left(q_{n}\right) \leq 1
$$

In other words, $q_{1} \oplus \cdots \oplus q_{n}$ is defined. From what was said before, it is clear that in general it is not satisfied for box models with more than 2 components.

The Local Orthogonality (LO) principle restricts set of states to those that do not violate any of Local Orthogonality inequalities. Furthermore, the $\mathrm{LO}^{\infty}$ principle is introduced $\underline{14}, \underline{24}$ that allows only such states on $k$-box model that when copied $n$ times do not violate any of Local Orthogonality inequalities in a $n k$-box model for an arbitrary $n$.

Our approach allows a dual look on this problem. Instead of restricting the set of states by the Local Orthogonality principle, we can extend the set of elements in the logic of a 
$k$-box model. In the simplest case we can generate a concrete orthomodular poset by $\mathcal{A}$ of Thm. 10. In this way we obtain a structure on which all states will satisfy all Local Orthogonality inequalities by definition.

Example 17 Consider a 3-box model consisting of $\mathfrak{B}_{\text {BIN }}$ boxes. A concrete orthomodular poset $\mathfrak{L}$ of subsets of $\Gamma_{\text {BIN }} \times \Gamma_{\text {BIN }} \times \Gamma_{\text {BIN }}$ generated by

$$
\mathcal{A}=\left\{a \times b \times c \mid a, b, c \in \operatorname{Atoms}\left(\mathfrak{B}_{\text {BIN }}\right)\right\}
$$

consists of 29142 elements and 192 atoms. All atoms of $\mathfrak{B}_{\text {BIN }}^{\otimes 3}$ are also atoms of $\mathfrak{L}$. Additional atoms were generated by complements of $\oplus$-sums of mutually disjoint elements that do not exists in the effect algebra $\mathfrak{B}_{\mathrm{BIN}}^{\otimes 3}$.

What is interesting, despite much richer set of atoms, the localized elements in $\mathfrak{L}$ and $\mathfrak{B}_{\text {BIN }}^{\otimes 3}$ are exactly the same. Thus we can regard $\mathfrak{L}$ as a different way of producing a composite 3-box system. We emphasize that in $\mathfrak{L}$ all LO inequalities are satisfied in any state, however we might expect that not all product states of components are admissible (cf. Ref. 14).

Additional propositions in $\mathfrak{L}$ can be interpreted as genuinely multi-box propositions (this should not pose any interpretational difficulties if we recall that in the quantum mechanics we have projectors onto entangled vectors).

This example indicates that the $\mathrm{LO}^{\infty}$ principle is equivalent to the statement that the proper way of producing the logic of composite $k$-box models is to generate an orthomodular poset instead of an effect algebra. That puts another restrictions on the set of states apart from no-signaling conditions (since there are more propositions, the set of states has to be smaller). States of subsystems have to be restricted accordingly so that all produce valid product states. The example also suggests that the quantum bound for correlations will not be attained even with $\mathrm{LO}^{\infty}$ principle, since we know that in the orthomodular poset it can be violated (c.f. the example of the 2-box model). However this remark is rather a hypothesis than a theorem: rigorous study of such kind of "orthoposet box-product" is required. 


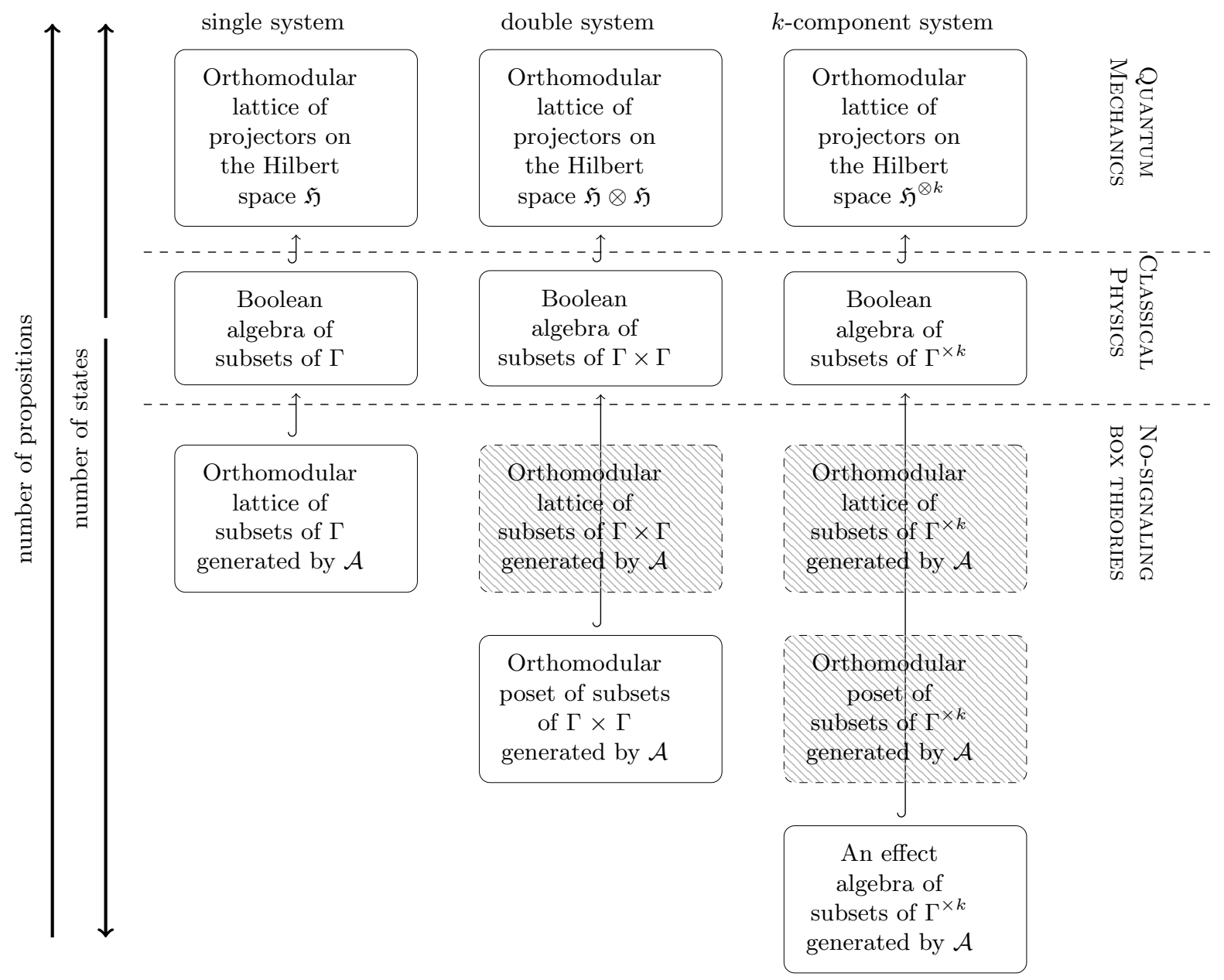

FIG. 2. Diagram shows how mathematical structures describing no-signaling box theories are related to structures of classical and quantum physics. Hooked arrows represent possibility of embedding. Arrows on the left indicate direction of increasing number of states and propositions. Dashed cells represent objects that can be constructed, however their properties where not yet studied. Notation is the same as in the Thm. 10.

\section{CONCLUSIONS}

Our results allows us to compare no-signaling box theories with classical and quantum theories using the same mathematical language. The results are summarized in the diagram 2. With no doubt no-signaling box theories posses properties that neither classical nor quantum mechanical systems do exhibit. However, detailed mathematical analysis reveals that the reason why they have such properties is completely different.

When we pass from classical to quantum theories, both numbers, of propositions and 
states, increase. One can think of quantum models (even such toy models as qubit, where canonical commutation relation cannot be realized) as an infinite (of continuum cardinality!) collections of classical models: one model for each maximal set of mutually commuting observables.

The multitude of propositions and states increases even more when we pass to composite systems. The tensor product of Hilbert spaces produces non-separable states and propositions. We need all states, both separable and non-separable, to determine the order in the set of all propositions ${ }^{29}$.

For no-signaling box theories the situation is dramatically different. We indeed get more states than in the classical case, but only because we decreased number of propositions describing our system. With increasing number of components our "ignorance", i.e. the number of propositions we declare to be non-verifiable, increases. This leads to orthomodular poset structure for arbitrary 2-box model and an effect algebra for a $k$-box model. While the first manifested itself in violation of CHSH-type inequality for 2-box models, the other results in violation of the Local Orthogonality principle ${ }^{14}$.

Moreover, the order of propositions in $\mathfrak{B}^{\otimes k}$ of $k$-box model is always determined by the classically correlated states, i.e. states obtained by restrictions of classical states on $\Gamma^{\times k}$ to $\mathfrak{B}^{\otimes k}$. This follows from the fact that all these structures are concrete.

Consequently, no-signaling box models are clearly not generalizations of quantum mechanics. While quantum mechanics generalizes classical in the "direction" of enrichment, no-signaling boxes generalize classical mechanics in the "direction" of impoverishment.

One can argue that if we take the set of states as a primary object, instead of the propositional system, we can still say that box models generalize quantum models because we have states that are not quantum. The are however two problems with this argument. Firstly, while it is obviously true that propositional system of a box system can be embedded into quantum mechanical model, the set of all states on a box model cannot. But neither we can embed in the opposite direction. We can map any quantum state on the state on a box model but this map is not injective (it is a restriction map).

Secondly, the state-based approach to physical theories is far more complicated than the observable-based one. The characterization of those convex sets that are set of states of operator algebras (thus establishing full equivalence of Schrödinger and Heisenberg pictures in quantum mechanics) was obtained quite recently 30,31 and is far from being trivial. Let 
us remark that (probably) the first serious steps in that direction were taken by Mielnik ${ }^{32}$ in 1974 but his program failed. This follows from the fact that the first-class properties of algebraic objects, whether these are operator algebras or quantum logic structures, manifests itself in very subtle geometrical properties of convex sets of states. Thus comparison of generality of theories on this level is rather complicated.

Finally, as was mentioned at the end of Sec. IV, our framework might be fruitful in the further investigations of the Local Orthogonality principle, since it shifts attention from states to sets of propositions that have more tractable structure.

\section{ACKNOWLEDGMENTS}

This work was done with the support of John Templeton Foundation grant. TT work was supported by the University of Gdańsk grant No. 538-5400-B295-16

\section{REFERENCES}

${ }^{1}$ S. Popescu and D. Rohrlich, "Quantum nonlocality as an axiom," Foundations of Physics 24, 379-385 (1994).

${ }^{2}$ A. Acín, N. Gisin, and L. Masanes, "From Bell's Theorem to Secure Quantum Key Distribution," Physical Review Letters 97, 120405 (2006).

${ }^{3}$ L. Masanes, A. Acín, and N. Gisin, "General properties of nonsignaling theories," Physical Review A 73, 1-9 (2006), arXiv:0508016 [quant-ph].

${ }^{4}$ J. Barrett, N. Linden, S. Massar, S. Pironio, S. Popescu, and D. Roberts, "Nonlocal correlations as an information-theoretic resource," Physical Review A 71, 22101 (2005).

${ }^{5}$ S. Pironio, A. Acín, S. Massar, A. B. de la Giroday, D. N. Matsukevich, P. Maunz, S. Olmschenk, D. Hayes, L. Luo, T. A. Manning, and C. Monroe, "Random numbers certified by Bell's theorem." Nature 464, 1021-4 (2010).

${ }^{6}$ H. Buhrman, R. Cleve, S. Massar, and R. De Wolf, "Nonlocality and communication complexity," Reviews of Modern Physics 82, 665-698 (2010).

${ }^{7}$ R. Gallego, L. Masanes, G. De La Torre, C. Dhara, L. Aolita, and A. Acín, "Full randomness from arbitrarily deterministic events." Nature Communications 4, 2654 (2013). ${ }^{8}$ G. Brassard, H. Buhrman, N. Linden, A. A. Méthot, A. Tapp, U. Falk, and F. Unger, 
"Limit on Nonlocality in Any World in Which Communication Complexity Is Not Trivial," Physical Review Letters 96, 250401 (2006).

${ }^{9}$ H. Buhrman, M. Christandl, F. Unger, S. Wehner, and A. Winter, "Implications of superstrong non-locality for cryptography," Proceedings of the Royal Society A: Mathematical, Physical and Engineering Sciences 462, 1919-19

${ }^{10}$ M. Pawłowski, T. Paterek, D. Kaszlikowski, V. Scarani, A. Winter, and M. Żukowski, "Information causality as a physical principle," Nature 461, 1101-1104 (2009).

${ }^{11}$ J. Allcock, N. Brunner, M. Pawlowski, and V. Scarani, "Recovering part of the boundary between quantum and nonquantum correlations from information causality," Physical Review A 80, 040103 (2009).

${ }^{12}$ L. Masanes and M. P. Müller, "A derivation of quantum theory from physical requirements," New Journal of Physics 13, 063001 (2011).

${ }^{13}$ J. Oppenheim and S. Wehner, "The uncertainty principle determines the nonlocality of quantum mechanics." Science (New York, N.Y.) 330, 1072-4 (2010).

${ }^{14}$ T. Fritz, A. B. Sainz, R. Augusiak, J. B. Brask, R. Chaves, A. Leverrier, and A. Acín, "Local orthogonality as a multipartite principle for quantum correlations." Nature Communications 4, 2263 (2013).

${ }^{15} \mathrm{M}$. Navascues and H. Wunderlich, "A glance beyond the quantum model," Proceedings of the Royal Society A: Mathematical, Physical and Engineering Sciences 466, 881-890

${ }^{16}$ H. Barnum, S. Beigi, S. Boixo, M. B. Elliott, and S. Wehner, "Local quantum measurement and no-signaling imply quantum correlations." Physical Review Letters 104, 140401 (2010).

${ }^{17}$ T. I. Tylec and M. Kuś, "Non-signaling boxes and quantum logics," Journal of Physics A 48, 505303 (2015).

${ }^{18}$ T. I. Tylec, M. Kuś, and J. Krajczok, "Non-signalling Theories and Generalized Probability," International Journal of Theoretical Physics 55, 3832-3842 (2016).

${ }^{19}$ T. I. Tylec and M. Kuś, "Tensor product of no-signalin boxes in the framework of quantum logic," Journal of Physics A , in print (2016).

${ }^{20}$ G. Birkhoff and J. Von Neumann, "The logic of quantum mechanics," Annals of mathematics , 823-843 (1936).

${ }^{21} \mathrm{G} . \mathrm{W}$. Mackey, The mathematical foundations of quantum mechanics (WA Benjamin (New York), 1963). 
${ }^{22}$ C. Piron, Foundations of quantum physics (Benjamin-Cummings Publishing Company, 1976) p. 123.

${ }^{23}$ G. Ludwig, Foundations of quantum mechanics, Volume 1 (Springer-Verlag, 1983) p. 426.

${ }^{24}$ A. B. Sainz, T. Fritz, R. Augusiak, J. B. Brask, R. Chaves, A. Leverrier, and A. Ac??n, "Exploring the local orthogonality principle," Physical Review A 89, 032117 (2014).

${ }^{25}$ A. Dvurecenskij and S. Pulmannová, New trends in quantum structures, Vol. 1 (2000).

${ }^{26} \mathrm{~W}$. Majewski and T. Tylec, "Remarks on Effect Algebras," International Journal of Theoretical Physics 49, 3185-3191 (2010).

${ }^{27}$ P. Pták and S. Pulmannová, Orthomodular Structures as Quantum Logics: Intrinsic Properties, State Space and Probabilistic Topics, Vol. 44 (Springer, 1991).

${ }^{28}$ M. L. Almeida, J. D. Bancal, N. Brunner, A. Acín, N. Gisin, and S. Pironio, "Guess your neighbor's input: A multipartite nonlocal game with no quantum advantage," Physical Review Letters 104, 230404 (2010).

${ }^{29}$ S. Pulmannová, "Tensor product of quantum logics," Journal of Mathematical Physics 26, 1-5 (1985).

${ }^{30}$ E. M. Alfsen and F. W. Shultz, Geometry of State Spaces of Operator Algebras (Springer Science \& Business Media, 2003).

${ }^{31}$ E. M. Alfsen and F. W. Shultz, State Spaces of Operator Algebras: Basic Theory, Orientations, and C (Springer Science \& Business Media, 2012).

${ }^{32}$ B. Mielnik, "Generalized quantum mechanics," Communications in Mathematical Physics 37, 221-256 (1974). 